Pacific Journal of Mathematics

REFLECTION AND APPROXIMATION BY INTERPOLATION
ALONG THE BOUNDARY FOR ANALYTIC FUNCTIONS 


\title{
REFLECTION AND APPROXIMATION BY INTERPOLATION ALONG THE BOUNDARY FOR ANALYTIC FUNCTIONS
}

\author{
JAMES M. SLOSS
}

Let there be given a function $f(z)$ analytic in an open connected set, not necessarily simply connected, which is bounded by simple closed analytic curves such that the function is continuous on the closure of the region and such that the real part of the function satisfies boundary conditions that are analytic in a neighborhood of the boundary. We want to interpolate $f(z)$ along the boundaries and find conditions that make the interpolants converge maximally to $f(z)$ throughout the closure of the region. The boundary condition on the real part of $f(z)$ permits the analytic continuation of $f(z)$ across the boundary curves and ensures that we are interpolating at points interior to the region of analyticity. In our error estimates (Theorem 1) maximal convergence depends in an essential way on how far we can reflect $f(z)$ and this in turn depends on the boundary values of the real part of $f^{\prime}(z)$ as well as on the geometry of the given region and its analytic boundaries. In Theorems 2 and 3 , a simply connected region is considered. Special points of interpolation are given, these depend only on the parametric representation of the boundary curves and not a conformal map. These points are the image points of the Chebysher polynomials.

Finally an example is given for a multiply connected region.

As is well known [2] Runge's beautiful theorem shows us that there exist certain "equidistributed" points on the analytic curves such that if we interpolate at these points the interpolants converge to the function. However, the proof depends on knowing the conformal map in order to know what the interpolation points are. Here we shall give conditions that do not require knowledge of the conformal map but for convergence depend on how far we can reflect. Along with these, we shall give simple error estimates. Moreover, we shall show that possible interpolation points are the images on the boundary of roots of the Chebyshev polynomials.

The aspects of this paper which are novel are

(i) the use of reflection

(ii) interpolation at boundary points which are gotten directly from the parametric representation of the boundary and do not depend on a conformal map

Received October 2, 1964. 
(iii) the use of the images of the roots of the Chebyshev polynomials as possible interpolation points.

Notation. Let $R$ be a connected set whose boundary is $\Gamma$. Let $\Gamma=\Gamma^{1} \cup \Gamma^{2} \cup \cdots \cup \Gamma^{s}$ where the $\Gamma^{j}$ are bounded analytic contours in the $z=x+i y$ plane given by $F^{j}(x, y)=0$ with $\left(F_{x}^{j}\right)^{2}+\left(F_{y}^{j}\right)^{2} \neq 0$ along $\Gamma^{j}, j=1,2, \cdots, s$, where the $F^{j}$ are real-valued analytic functions of $x$ and $y$. We assume further that the $\Gamma^{j}$ are pairwise disjoint. Let $\Gamma^{1}$ contain in its interior $\Gamma^{2}, \Gamma^{3}, \cdots, \Gamma^{s}$ and contain in its exterior the point at infinity. Let $\Gamma^{j}$ contain in its interior $a_{j} 2 \leqq j \leqq s$. As shown in [3] there are "reflection" functions $G_{j}(z)$ defined on a neighborhood $D^{j} \cup \Gamma^{j} \cup \hat{D}^{j}$ of $\Gamma^{j}$. Assume $G_{j}(z)$ single-valved on $D^{j} \cup \Gamma^{j} \cup \hat{D}^{j}[3]$ shows.

(1) $z=\overline{G_{j}(z)}$ is $\Gamma^{j}$.

(2) $G_{j}(z)$ is analytic on $D^{j} \cup \Gamma^{j} \cup \hat{D}^{j}$, where $D^{j}$ is contained in the connected $R$ and $D^{j}$ is contained in the complement of $\Gamma^{j} \cup D^{j}$ for $j=1,2, \cdots, s$.

(3) The transformation $\hat{z}=\overline{G(z)}$ is an involution; i.e. $\hat{\hat{z}}=z$.

(4) If $z$ is in $D^{j}$ then $\hat{z}$ is in $\hat{D}^{j}$ and if $z$ is in $\hat{D}^{j}$ then $\hat{z}$ is in $D^{j}$.

(5) $\overline{G\left[D^{j}\right]}=\hat{D}^{j}$ and $\overline{G\left[\hat{D}^{j}\right]}=D^{j}$. We assume the boundary of $\hat{D}^{j}$, that is not $\Gamma^{j}$, is a contour $C^{j}$ and $G_{j}(z)$ is continuous on $\hat{D}^{j} \cup C^{j}$.

THEOREM 1. (H 1) Let $f(z)$ be an analytic single valued function on $R$ whose boundary is $\Gamma$ such that the real part of $f(z)$ solves the Dirichlet problem in $R$ with real boundary values $B_{j}(z)$ on $\Gamma^{j}$ where $B_{j}(z)$ are single-valued and continuous in $D^{j} \cup \Gamma^{j} \cup \hat{D}^{j} \cup C^{j}$ and analytic in $D^{j} \cup \Gamma^{j} \cup \hat{D}^{j}$. Let $f(z)$ be continuous and single-valued on $R \cup \Gamma$.

(H. 2) Let $z_{n_{1} 1}^{j}, z_{n_{j} 2}^{j}, \cdots, z_{n_{n} n^{+1}}^{j}, n_{j}=0,1,2, \cdots$, be points of $\Gamma^{j}, j=1,2, \cdots, s$. Let $p_{n_{1}}^{j}(z)$ be the polynomial in $z$ of degree $n_{1}$ that agrees with $B_{1}(z)$ at $z_{n_{1}}^{1}, z_{n_{1}}^{1}, \cdots, z_{n_{1} n_{1}+1}^{1}$ and let $p_{n_{j}}^{j}(z)(2 \leqq j \leqq s)$ be the polynomial in $1 /\left(z-a_{j}\right)$ that agrees with $B_{j}(z)$ for $z-a_{j}=z_{n_{j} 1}^{j}$, $z_{n_{2}}^{j}, \cdots, z_{n_{n+1}}^{j}$ where $a_{j}$ is a point inside $c^{j}$.

(H. 3) Let

$$
\begin{aligned}
& \delta_{n_{1}}^{1}=\min _{t \text { on } \sigma^{1}} \prod_{k=1}^{n_{1}+1}\left|t-z_{n_{1} k}^{1}\right| \\
& \mu_{n_{1}}^{1}=\max _{z \text { on } \Gamma^{1}} \prod_{k=1}^{n_{1}+1}\left|z-z_{n_{1} k}^{1}\right|
\end{aligned}
$$

and

$$
\begin{aligned}
& \delta_{n_{j}}^{j}=\min _{t \text { on } C^{j}} \prod_{k=1}^{n_{j}+1}\left|\frac{t-a_{j}-z_{n^{k} k}^{j}}{t-a_{j}}\right|, 2 \leqq j \leqq s \\
& \mu_{n_{j}}^{j}=\max _{z \text { on } \Gamma^{j}} \prod_{k=1}^{n_{j}+1}\left|\frac{z-a_{j}-z_{n^{k} k}^{j}}{z-a_{j}}\right|, 2 \leqq j \leqq s .
\end{aligned}
$$


(H. 4) Let $\mu_{n_{j}}^{j} / \delta_{n_{g}}^{j} \rightarrow 0$ as $n_{j} \rightarrow \infty, j=1,2, \cdots, s$. Then for $\mu=\left(\frac{1}{n_{1}}, \frac{1}{n_{2}}, \cdots, \frac{1}{n_{s}}\right)$ and $|\mu|=\max \left\{\frac{1}{n_{1}}, \frac{1}{n_{2}}, \cdots, \frac{1}{n_{s}}\right\}$.

(C. 1) $R_{\mu}(z)=\sum_{j=1}^{s} p_{n_{j}}^{j}(z)$ converges uniformly to $f(z)$ in $R \cup \Gamma$ as $|\mu| \rightarrow 0$ and thus $R e R_{\mu}(z)$ converges uniformly to $u(x, y)$ in $R$ and uniformly to $B_{j}(z)$ on $\Gamma^{j}$.

(C. 2) Moreover in $R \cup \Gamma$ :

$$
\left|f(z)-R_{\mu}(z)\right| \leqq \frac{1}{2 \pi} \sum_{j=1}^{s} \frac{L_{j} M_{j}}{\delta_{j}} \mu_{n_{j}}^{j} / \delta_{n_{j}}^{j}
$$

where $L_{j}=$ length of $C^{j}, M_{j}=\max _{t \text { on } 0^{j}}|f(t)|$ and $\delta_{j}=\inf _{z \text { on } \Gamma^{j}} \min _{t \text { on } \sigma^{j}}|t-z|$.

Proof. In order to avoid notation that only confuses, we shall prove the theorem for the case $s=2$.

We first analytically continue $f(z)$ into $R \cup \Gamma^{1} \cup \hat{D}^{1} \cup \Gamma^{2} \cup \hat{D}^{2}$. Let $f_{j}^{*}(z)=\overline{f\left[\overline{G_{j}(z)}\right]}$ for $z$ in $\Gamma^{j} \cup \hat{D}^{j} . f_{j}^{*}(z)$ is defined and analytic for $z$ in $\Gamma^{j} \cup \hat{D}^{j}$ since $\hat{z}=\overline{G_{j}(z)}$ is in $D^{j}$ for $z$ in $\hat{D}^{j}$ and $G_{j}(z)$ is analytic for $z$ in $\Gamma^{j} \cup \hat{D}^{j}$. But $f_{j}^{*}(z)=\overline{f(z)}$ for $z$ on $\Gamma^{j}$, thus on $\Gamma^{j}$

$$
f(z)+f_{j}^{*}(z)=2 B_{j}(z) .
$$

Thus $f(z)=2 B_{j}(z)-f_{j}^{*}(z)$ analytically continues $f(z)$ into $\Gamma^{j} \cup \hat{D}^{j}$ since $f(z)$ is continuous up to and on $\Gamma^{j}$. Moreover, $f(z)$ is continuous on

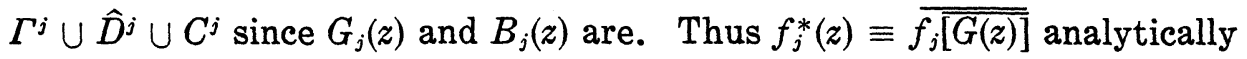
continues $f^{*}(z)$ into $\Gamma^{j} \cup D^{j}$ since $f_{j}^{*}(z)$ is continuous up to and on $\Gamma^{j}$. Let $\alpha_{n+1}(z)=\left(z-z_{n 1}^{1}\right)\left(z-z_{n 2}^{1}\right) \cdots\left(z-z_{n n+1}^{1}\right)$

$$
\beta_{m+1}(z)=\left(\frac{1}{z-a_{2}}-\frac{1}{z_{m 1}^{2}}\right)\left(\frac{1}{z-a_{2}}-\frac{1}{z_{m 2}^{2}}\right) \cdots\left(\frac{1}{z-a_{2}}-\frac{1}{z_{m m+1}^{2}}\right) .
$$

Then for $z$ on $\Gamma$ :

$$
\begin{aligned}
p_{n m}(z)= & \frac{1}{2 \pi i} \oint_{\sigma^{1}} \frac{f(t)}{t-z} \frac{\alpha_{n+1}(t)-\alpha_{n+1}(z)}{\alpha_{n+1}(t)} d t \\
& -\frac{1}{2 \pi i} \oint_{o^{2}} \frac{f(t)}{t-z} \frac{\beta_{m+1}(t)-\beta_{m+1}(z)}{\beta_{m+1}(t)} d t
\end{aligned}
$$

where $p_{n m}(z)$ is a rational function of $z, p_{n m}(z)=p_{n}^{1}(z)+p_{m+1}^{2}(z)$ in which $p_{n}^{1}(z)$ is the polynomial in $z$ of degree $\leqq n$ got by interpolating $f(z)$ along $\Gamma^{1}$ at $z_{n 1}^{1}, z_{n 2}^{1}, \cdots, z_{n n+1}^{1}$ and $p_{m+1}^{2}(z)$ is the polynomial of degree $m+1$ in $1 /\left(z-a_{2}\right)$ got by interpolating $f(z)$ along $\Gamma^{2}$ so that $p_{m+1}^{2}\left(a_{2}+z_{m j}^{2}\right)=f\left(z_{m j}^{2}\right)$. To see the latter let $x=1 /\left(z-a_{2}\right)$ and $y=$ 
$1 /\left(t-a_{2}\right)$ then $\beta_{m+1}(t)=b_{m+1}(y)$ where $b_{m+1}(y)$ is a monic polynomial in $y$ of degree $\leqq m+1$, thus we have

$$
\beta_{m+1}(t)-\beta_{m+1}(z)=b_{m+1}(x)-b_{m+1}(y)=(x-y) \sum_{i=1}^{m} a_{i}(x) y^{i}
$$

where $a_{i}(x)$ are polynomials in $x$ of degree $\leqq m$. But

$$
x-y=\frac{1}{z-a_{2}}-\frac{1}{t-a_{2}}=(t-z) x y
$$

thus

$$
\int_{0^{2}} \frac{f(t)}{t-z} \frac{\beta_{m+1}(t)-\beta_{m+1}(z)}{\beta_{m+1}(t)} d t
$$

is a polynomial of degree $\leqq m+1$ in $1 /\left(z-a_{2}\right)$. The error for $z$ on $\Gamma$ is given by:

$$
f(z)-p_{n m}(z)=\frac{1}{2 \pi i} \int_{O^{1}} \frac{f(t)}{t-z} \frac{\alpha_{n+1}(z)}{\alpha_{n+1}(t)} d t-\frac{1}{2 \pi i} \int_{o^{2}} \frac{f(t)}{t-z} \frac{\beta_{m+1}(z)}{\beta_{m+1}(t)} d t .
$$

Note that:

$$
\begin{aligned}
\left|\alpha_{n+1}(z)\right| \leqq & \mu_{n}^{1},\left|\alpha_{n+1}(t)\right| \geqq \delta_{n}^{1} \text { for } z \text { on } \Gamma^{1} \text { and } t \text { on } C^{1} \text { and: } \\
& \frac{\frac{1}{z-a_{2}}-\frac{1}{z_{n 1}^{2}}}{\frac{1}{t-a_{2}}-\frac{1}{z_{n 1}^{2}}}=\frac{z-a_{2}-z_{n 1}^{2}}{t-a_{2}-z_{n 1}^{2}} \frac{t-a_{2}}{z-a_{2}}
\end{aligned}
$$

and thus

$$
\left|\frac{\beta_{m+1}(z)}{\beta_{m+1}(t)}\right| \leqq \frac{\mu_{m}^{2}}{\delta_{m}^{2}} \text { for } z \text { on } \Gamma^{2} \text { and } t \text { on } C^{2}
$$

From these it follows:

$$
\left|f(z)-p_{n m}(z)\right| \leqq \frac{1}{2 \pi}\left\{\frac{L_{1} M_{1}}{\delta_{1}} \frac{\mu_{n}^{1}}{\delta_{1}}+\frac{L_{2} M_{2}}{\delta_{2}} \frac{\mu_{m}^{2}}{\delta_{m}^{2}}\right\} \quad \text { for } z \text { on } \Gamma
$$

where $L_{j}$ is the length of $C^{j}$,

$$
M_{j}=\max _{t \text { on } c^{j}}|f(t)|, \quad \text { and } \delta_{j}=\inf _{z \text { on } \Gamma^{j}} \min _{t \text { on } 0^{j}}|t-z|
$$

which is the result.

We next consider the case when $\Gamma$ is a single analytic contour and $\left(C^{j}=C\right)$ we write $\Gamma$ in parametric form as $z(\sigma)=x(\sigma)+i y(\sigma)$ where $-1 \leqq \sigma \leqq 1$. Let $\left|z\left(\sigma_{2}\right)-z\left(\sigma_{1}\right)\right| \leqq A\left|\sigma_{2}-\sigma_{1}\right|$, let $\Gamma$ contain the origin and 
THEOREM 2 (H. 1) Let $f(z)$ be an analytic single-valued function on $R$ whose boundary is $\Gamma$ such that the real part of $f(z)$ solves the Dirichlet problem in $R$ with real boundary values $B(z)$ on $\Gamma$ where $B(z)$ is a single-valued analytic function on $D \cup \Gamma \cup \hat{D}$ continuous on $\Gamma \cup \hat{D} \cup C$. Let $f(z)$ be continuous and single-valued on $R \cup \Gamma$.

(H. 2) Let $z_{j}^{n}=z\left(\sigma_{j}^{n}\right)$ where

$$
\sigma_{j}^{n}=\cos [(2 j-1) \pi /(2 n+2)], j=1,2, \cdots, n+1
$$

(H. 3) $\delta=\inf _{z \text { on } \Gamma \text { in } 0} \min _{t}|t-z|$

\section{Then}

(H. 4) $A<2 \delta$.

(C. 1) $p_{n}(z)=\sum_{j=1}^{n+1} f\left(z_{j}^{n}\right) \frac{\omega_{n+1}(z)}{\omega_{n+1}^{\prime}\left(z_{j}^{n}\right)\left(z-z_{j}^{n}\right)}$, where

$\omega_{n+1}(z)=\prod_{k=1}^{n+1}\left(z-z_{k}^{n}\right)$ and prime denotes differentiation, converges uniformly to $f(z)$ on $R \cup \Gamma$ as $n \rightarrow \infty$

(C. 2) $\left|f(z)-p_{n}(z)\right| \leqq \frac{L M}{\pi \delta}\left(\frac{A}{2 \delta}\right)^{n+1}$

where $M$ is a constant depending on $f, L$ is length of $\Gamma$.

Proof. As in the proof of Theorem 1 we have for $z$ on $\Gamma$

$$
\left|f(z)-p_{n}(z)\right| \leqq \frac{L}{2 \pi} M \max _{z \text { on } \Gamma}\left|\omega_{n+1}(z)\right| / \delta^{n+2} .
$$

But

$$
\begin{aligned}
\left|\omega_{n+1}(z)\right| & =\left|\left(z-z_{1}^{n}\right)\left(z-z_{2}^{n}\right) \cdots\left(z-z_{n+1}^{n}\right)\right| \\
& \leqq A^{n+1}\left|\left(\sigma-\sigma_{1}^{n}\right)\left(\sigma-\sigma_{2}^{n}\right) \cdots\left(\sigma-\sigma_{n+1}^{n}\right)\right|
\end{aligned}
$$

where the $\sigma_{j}^{n}$ are the roots of the Chebyshev polynomial

$$
T_{n+1}(\sigma)=\cos [(n+1) \operatorname{arc} \cos \sigma]
$$

of degree $n+1$. Thus since $\left(\sigma-\sigma_{1}^{n}\right)\left(\sigma-\sigma_{2}^{n}\right) \cdots\left(\sigma-\sigma_{n+1}^{n}\right)$ is monic

$$
\left|\omega_{n+1}(z)\right| \leqq A^{n+1} T_{n+1}(\sigma) / 2^{n}
$$

Thus

$$
\max _{z \text { on } \Gamma}\left|\omega_{n+1}(z)\right| \leqq A^{n+1} / 2^{n} \quad \text { and } \quad\left|f(z)-p_{n}(z)\right| \leqq \frac{L M}{\delta \pi}\left(\frac{A}{2 \delta}\right)^{n+1} .
$$

Next let $\Gamma: z(s)=x(s)+i y(s)$ where $s$ is arc length $0 \leqq s \leqq L$.

Theorem 3. (H. 1) Same as Theorem 2.

(H. 2) Same as Theorem 2 but $z_{j}^{n}=z\left(s_{j}^{n}\right)$ where 


$$
s_{j}^{n}=\frac{L}{2} \cos [(2 j-1) \pi / 2(n+1)]+\frac{L}{2}, j=1,2, \cdots, n+1 .
$$

(H 3) $\delta=\inf _{z \text { on } r} \min _{t \text { on } 0}|t-z|$.

(H 4) $L<4 \delta$.

(C 1) Same as Theorem 2.

(C 2) $\left|f(z)-p_{n}(z)\right| \leqq \frac{L M}{\pi \delta}\left(\frac{L}{4 \delta}\right)^{n+1}$

where $M$ is a constant depending on $f$.

Proof. As in the proof of theorem for $z$ on $\Gamma$ :

$$
\left|f(z)-p_{n}(z)\right| \leqq \frac{L}{2 \pi} M \max \left|\omega_{n+1}(z)\right| / \delta^{n+2} \text {. }
$$

But since $\left|z-z_{j}^{n}\right| \leqq\left|s-s_{j}^{n}\right|$ where $z=z(s)$ and $z_{j}^{n}=z\left(s_{j}^{n}\right)$ and since $\left|\left(s-s_{1}^{n}\right)\left(s-s_{2}^{n}\right) \cdots\left(s-s_{n+1}^{n}\right)\right| \leqq L^{n+1} / 2^{2 n+1}$ see e.g. [1] we have

$$
\left|f(z)-p_{n}(z)\right| \leqq \frac{L M}{\pi \delta}\left(\frac{L}{4 \delta}\right)^{n+1}
$$

EXAMPLE. We shall now apply the ideas of this paper to a particular geometrical configuration. Let

$\Gamma^{1}$ be a circle of radius 15 centered at the origin

$\Gamma^{2}$ be a circle of radius 1 centered at $(-13,0)$

$\Gamma^{3}$ be an ellipse

$$
\frac{x^{2}}{a^{2}}+\frac{y^{2}}{b^{2}}=1, a=1.075, b=1 .
$$

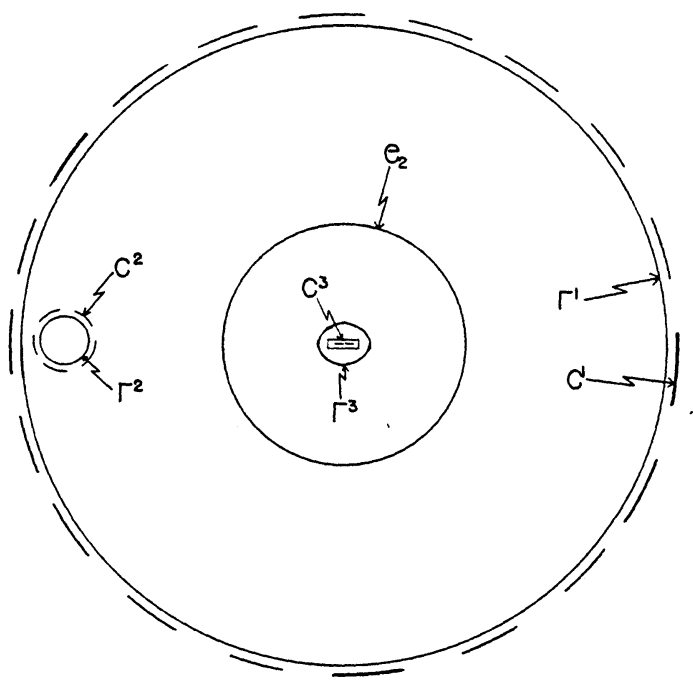

Fig. 1. 
Let $R$ be the interior of $\Gamma^{1}$ less $\Gamma^{2}, \Gamma^{3}$ and the interiors of $\Gamma^{2}$ and $\Gamma^{3}$. Let $f(z)$ be analytic on $R$ and continuous on $R \cup \Gamma^{1} \cup \Gamma^{2} \cup \Gamma^{3}$. Moreover let the real part of $f(z)$ satisfy boundary conditions $B_{1}(z)$ on $\Gamma^{1}, B_{2}(z)$ on $\Gamma^{2}$ and $B_{3}(z)$ on $\Gamma^{3}$ where:

$B_{1}(z)$ is analytic on $|z|=15$

$B_{2}(z)$ is analytic on $|z+13|=1$

and $B_{3}(z)$ is analytic in and on $\Gamma^{3}-\{-.395<x<.395, y=0\}$ See figure.

For example we might have $\operatorname{Re} f(z)=P_{k}(x, y)$ on $\Gamma^{k}(k=1,2,3)$ where $P_{k}(x, y)$ is a polynomial.

Then since:

$$
\begin{aligned}
& G_{1}(z)=(15)^{2} z^{-1} \\
& G_{2}(z)=(z+13)^{-1}-13
\end{aligned}
$$

(E. 1)

$$
G_{3}(z)=\frac{z\left(a^{2}+b^{2}\right) \pm 2 a b \sqrt{z^{2}+b^{2}-a^{2}}}{a^{2}-b^{2}} a>b \text { see [3] }
$$

and $z=\hat{z}=\overline{G_{k}(z)}$ on $\Gamma_{k}$ we have on $\Gamma_{k}$

$$
P_{k}\left(\frac{z+\bar{z}}{2}, \frac{z-\bar{z}}{2 i}\right)=P_{k}\left[\frac{z+G_{k}(z)}{2}, \frac{z-G_{k}(z)}{2 i}\right] \equiv B_{k}(z)
$$

which are meromorphic functions that fulfill the requirements of $B_{1}(z), B_{2}(z)$ and $B_{3}(z)$ (in the case of $B_{3}(z)$ we make a cut between the foci $\pm \sqrt{\left.a^{2}-b^{2}\right)}$.

Let

$$
r_{k}=15 \exp \left(\frac{2 \pi i}{n+1} k\right) \text { and } \alpha_{n+1}(z)=\left(z-r_{1}\right)\left(z-r_{2}\right) \cdots\left(z-r_{n+1}\right)
$$

and

$$
p_{n}(z)=\sum_{k=1}^{n+1} f\left(r_{k}\right) \frac{\alpha_{n+1}(z)}{\alpha_{n+1}\left(r_{k}\right)\left(z-r_{k}\right)}
$$

where the prime signifies differentiation. $p_{n}(z)$ is clearly the polynomial of degree $\leqq n$ that interpolates $f(z)$ at $z=r_{k}$ on $\Gamma^{1}, k=1, \cdots, n+1$. Next let

$$
s_{k}=\exp \left(\frac{2 \pi i}{m+1} k\right)
$$

and

$$
\beta_{m+1}(z)=\left(\frac{1}{z+13}-\frac{1}{s_{1}}\right)\left(\frac{1}{z+13}-\frac{1}{s_{2}}\right) \cdots\left(\frac{1}{z+13}-\frac{1}{s_{m+1}}\right)
$$

and 


$$
q_{m}(z)=\sum_{k=1}^{m+1} f\left(s_{k}-13\right) \frac{\beta_{m+1}(z)}{B_{m+1}^{\prime}\left(s_{k}-13\right)\left(\frac{1}{s_{k}}-\frac{1}{z+13}\right)\left(s_{k}\right)^{2}}
$$

$q_{m}(z)$ is a polynomial of degree $\leqq m$ in $1 /(z+13)$ such that $q_{m}\left(s_{k}-13\right)=$ $f\left(s_{k}-13\right)$ where $s_{k}-13$ is on $\Gamma^{2}, k=1,2, \cdots, m+1$.

Finally let $l$ be the length of the ellipse $\Gamma^{3}$ and

$$
\sigma_{k}=\cos [(2 k-1) \pi / 2(j+1)], k=1,2, \cdots, j+1 .
$$

Then the ellipse $\Gamma^{3}$ can be written

$$
z(\sigma)=x(\sigma)+i y(\sigma)=a \cos (2 \pi \sigma / l)+i b \sin (2 \pi \sigma / l),-l / 2 \leqq \sigma \leqq l / 2
$$

$\sigma$ is are length parameter shifted. Let

$$
t_{k}=z\left(\sigma_{k} l / 2\right) \quad \text { and } \quad \kappa_{j+1}(z)=\left(\frac{1}{z}-\frac{1}{t_{1}}\right)\left(\frac{1}{z}-\frac{1}{t_{2}}\right) \cdots\left(\frac{1}{z}-\frac{1}{t_{j+1}}\right)
$$

and

$$
r_{j}(z)=\sum_{k=1}^{j+1} f\left(t_{k}\right) \frac{\kappa_{j+1}(z)}{\kappa_{j+1}^{\prime}\left(t_{k}\right)\left(\frac{1}{t_{k}}-\frac{1}{z}\right)\left(t_{k}\right)^{2}}
$$

$r_{j}(z)$ is clearly the polynomial in $1 / z$ of degree $\leqq j$ such that $r_{j}\left(t_{k}\right)=$ $f\left(t_{k}\right) k=1,2, \cdots, j+1$ where $t_{k}$ is on $\Gamma^{3}$.

Then the assertion is

$$
p_{n}(z)+q_{m}(z)+r_{j}(z)
$$

converges uniformly to $f(z)$ on $R \cup \Gamma^{1} \cup \Gamma^{2} \cup \Gamma^{3}$ as

$$
\frac{1}{n}+\frac{1}{m}+\frac{1}{j} \rightarrow 0 \text {. }
$$

For $\Gamma^{1}$, we use Runge's theorem. Since $B_{1}(z)$ is analytic on $\Gamma^{1}$, then $f(z)$ can be continued across $\Gamma^{1}$, i.e. $f(z)$ is analytic for $15 \leqq$ $|z| \leqq 15+\varepsilon$ where $\varepsilon$ is some positive number. Thus in the notation of the theorem

$$
\begin{aligned}
\delta_{n}^{1} & =\min _{|t|=15+\varepsilon}\left|\prod_{k=1}^{n+1}\left(t-r_{k}\right)\right|=\min _{|t|=15+\varepsilon}\left|t^{n+1}-15^{n+1}\right| \\
& =\min _{|\tau|=1+\varepsilon / 15} 15^{n+1}\left|\tau^{n+1}-1\right| \geqq 15^{n+1} \min _{|\tau|=1+\varepsilon / 15}\left\{|\tau|^{n+1}-1\right\} \\
& =15^{n+1}\left\{[1+\varepsilon / 15]^{n+1}-1\right\} \\
\mu_{n}^{1} & =\max _{|z|=15}\left|\prod_{k=1}^{n+1}\left(z-r_{k}\right)\right|=15^{n+1} \max _{|\zeta|=1}\left|\zeta^{n+1}-1\right| \leqq 2 \cdot 15^{n+1}
\end{aligned}
$$

and 
(E 2) $u_{n}^{1} / \delta_{n}^{1} \leqq 2 /\left\{[1+\varepsilon / 15]^{n+1}-1\right\} \rightarrow 0$ as $n \rightarrow \infty$.

For $\Gamma^{2}$, since $B_{2}(z)$ is analytic on $\Gamma^{2}$, then $f(z)$ can be continued across $\Gamma^{2}$, i.e. $f(z)$ is analytic for $1-\varepsilon \leqq|z+13| \leqq 1$ where $\varepsilon$ is some positive number. Thus if $C^{2}=\{z:|z+13|=1-\varepsilon\}$ we have:

$$
\begin{aligned}
\max _{z \text { on } \Gamma^{2}}\left|\beta_{m+1}(z)\right| & =\max _{z \text { on } \Gamma^{2}}\left|\prod_{k=1}^{m+1}\left(\frac{1}{z+13}-\frac{1}{s_{k}}\right)\right|=\max _{z \text { on } \Gamma^{2}}\left|\left(\frac{1}{z+13}\right)^{m+1}-1\right| \\
& =\max _{|\zeta|=1}\left|\left(\frac{1}{\zeta}\right)^{m+1}-1\right| \leqq 2
\end{aligned}
$$

and

$$
\begin{aligned}
\min _{t \text { on } O^{2}}\left|\beta_{m+1}(t)\right| & =\min _{t \text { on } \sigma^{2}}\left|\left(\frac{1}{t+13}\right)^{m+1}-1\right| \\
& =\min _{|\zeta|=1-\varepsilon}\left|\left(\frac{1}{\zeta}\right)^{m+1}-1\right| \geqq\left(\frac{1}{1-\varepsilon}\right)^{m+1}-1 .
\end{aligned}
$$

From these we see that

(E. 3)

$$
\frac{\max _{z \text { on } \Gamma^{2}}\left|\beta_{m+1}(z)\right|}{\min _{t \text { on } \sigma^{2}}\left|\beta_{m+1}(t)\right|} \leqq \frac{2}{\left(\frac{1}{1-\varepsilon}\right)^{m+1}-1} \rightarrow 0 \text { as } m \rightarrow \infty,
$$

For $\Gamma^{3}$ we note from the reflection function $G(z)$ given by (E. 1) that the interior of the ellipse $\Gamma^{3}$ minus the line $-c \leqq x \leqq c, c^{2}=a^{2}-b^{2}$, is reflected exterior to the given ellipse but interior to the ellipse $e_{2}$

$$
\frac{\hat{x}^{2}}{\hat{a}^{2}}+\frac{\hat{y}^{2}}{\hat{b}^{2}}=1
$$

where $\hat{a}=\left(a^{2}+b^{2}\right) / c, \hat{b}=2 a b / c$.

In the case of our ellipse we have $a=1.075, b=1$ and $c=.395$, $\hat{a}=5.46, \hat{b}=5.44$, thus $e_{2}$ is contained in $\Gamma^{1}$, and does not intersect or contain points of $\Gamma^{2}$ and thus $f(z)$ can be extended to be analytic in $\Gamma^{3}-\{z \mid-.395<x<.395, y=0\}$.

The length of the ellipse $\Gamma^{3}$ is given by:

$$
l=4 a \int_{0}^{\pi / 2} \sqrt{1-k^{2} \sin ^{2} \theta d \theta}
$$

where

$$
\begin{aligned}
& k=c / a<1 . \text { In our case } k=.368 \text { and thus } \\
& l=4 a(1.516)
\end{aligned}
$$

using a table for elliptic integrals. Let $c^{3}$ be the rectangular contour 


$$
\begin{aligned}
& (-.395-\varepsilon \leqq x \leqq .395+\varepsilon, y=-\varepsilon),(x=.395+\varepsilon,-\varepsilon \leqq y \leqq \varepsilon) \\
& (-.395-\varepsilon \leqq x \leqq .395+\varepsilon, y=\varepsilon),(x=-.395-\varepsilon,-\varepsilon \leqq y \leqq \varepsilon)
\end{aligned}
$$

where $\varepsilon>0$ is arbitrarily small. Then consider

$$
\frac{1}{2 \pi i} \int_{\sigma^{3}} \frac{f(t)}{t-z} \frac{\kappa_{j+1}(z)}{\kappa_{j+1}(t)} d t
$$

But

$$
\begin{aligned}
\left|\kappa_{j+1}(z)\right| & =\left|\prod_{k=1}^{j+1}\left(\frac{1}{z}-\frac{1}{t_{k}}\right)\right|=\left|\prod_{k=1}^{j+1} \frac{t_{k}-z}{t_{k} z}\right| \\
& \leqq \prod_{k=1}^{j+1} \frac{\sigma-\sigma_{k} l / 2}{\left|t_{k}\right||z|} \leqq\left(\frac{l}{2}\right)^{j+1} \prod_{k=1}^{j+1} \frac{\left|\theta-\sigma_{k}\right|}{\left|t_{k}\right|} \cdot \sigma=\text { arc length, }
\end{aligned}
$$

where $-1 \leqq \theta \leqq 1$ since $|z| \geqq 1$ for $z$ on $\Gamma^{3}$. Also for $t$ on $C^{3}$

$$
\left|\kappa_{j+1}(t)\right|=\left|\prod_{k=1}^{j+1} \frac{t_{k}-t}{t_{k} t}\right| \geqq(a-c-\eta \varepsilon)^{j+1} \prod_{k=1}^{j+1} \frac{1}{\left|t_{k}\right||t|}
$$

where $\eta$ is some fixed constant. But since $|t| \leqq c+\varepsilon / 2$ for $t$ on $c^{3}$ we see that

$$
\left|\kappa_{j+1}(t)\right| \geqq\left(\frac{a-c-\eta \varepsilon}{c+\varepsilon \sqrt{2}}\right)^{j+1} \prod_{k=1}^{j+1} \frac{1}{\left|t_{k}\right|}
$$

Combining the above results gives

$$
A \equiv\left|\kappa_{j+1}(z) / \kappa_{j+1}(t)\right| \leqq\left(\frac{c+\varepsilon \sqrt{2}}{a-c-\eta \varepsilon}\right)^{j+1}\left(\frac{l}{2}\right)^{j+1} 2^{-j}\left|T_{j+1}(\theta)\right|
$$

where we have utilized the fact that the $\sigma_{k}$ are the roots of the Chebyshev polynomial $T_{j+1}(\theta)=\operatorname{cox}[(j+1)$ are $\cos \theta]$. Thus

$$
A \leqq 2\left(\frac{c+\varepsilon \sqrt{2}}{a-c-\eta \varepsilon}\right)^{j+1}\left(\frac{l}{4}\right)^{j+1} \text {. }
$$

But

$$
\frac{l}{4 a}=1.516<\frac{1}{a} \frac{a-c-\eta \varepsilon}{c+\varepsilon \sqrt{2}}=\frac{1}{1.075} \frac{.680-\eta \varepsilon}{.395+\sqrt{2 \varepsilon}},=1.60+g(\varepsilon)
$$

where $g(\varepsilon) \rightarrow 0$ as $\varepsilon \rightarrow 0$. Thus for $\varepsilon$ sufficiently small

$$
l / 4 \leqq \frac{a-c-\eta \varepsilon}{c+\varepsilon \sqrt{2}}
$$

Utilizing (E. 2), (E. 3) and (E. 4) we have from Theorem 1 that $p_{n}(z)+p_{m}(z)+r_{j}(z)$ converges uniformly to $f(z)$ in $R \cup \Gamma^{1} \cup \Gamma^{2} \cup \Gamma^{3}$ as $(1 / n)+(1 / m)+(1 / j) \rightarrow 0$. 
We remark finally that there would be no new difficulties if $\Gamma$ had contained in addition $\Gamma^{4} \cup \Gamma^{5} \cup \Gamma^{6}$ where $\Gamma^{4}$ is the circle $|z-10 i|=4$, $\Gamma^{5}$ the circle $|z+10 i|=4$ and $\Gamma^{6}$ is the circle $|z-12|=2$.

\section{REFERENCES}

1. T. M. Apostol, Calculus, Vol. II, Blaisdell Pub. Co.

2. S. N. Mergelyan, Uniform approximations to functions of a complex variable, Trans. Ser. 1, Vol. 3, Series and Approximation, Amer. Math. Soc.

3. J. M. Sloss, Reflection of Biharmonic Functions Across Analytic Boundary Conditions with Examples, Pacific. J. Math. 13 (1963), 1401-1415. 



\section{PACIFIC JOURNAL OF MATHEMATICS}

\section{EDITORS}

\author{
H. SAMmLSON \\ Stanford University \\ Stanford, California \\ R. M. BLUMENTHAL \\ University of Washington \\ Seattle, Washington 98105
}

\author{
*J. DUGUNDJI \\ University of Southern California \\ Los Angeles, California 90007
}

RICHARD ARENS

University of California

Los Angeles, California 90024

\section{E. F. BECKENBACH \\ B. H. NEUMANN \\ ASSOCIATE EDITORS}

\section{SUPPORTING INSTITUTIONS}

\author{
UNIVERSITY OF BRITISH COLUMBIA \\ CALIFORNIA INSTITUTE OF TECHNOLOGY \\ UNIVERSITY OF CALIFORNIA \\ MONTANA STATE UNIVERSITY \\ UNIVERSITY OF NEVADA \\ NEW MEXICO STATE UNIVERSITY \\ OREGON STATE UNIVERSITY \\ UNIVERSITY OF OREGON \\ OSAKA UNIVERSITY \\ UNIVERSITY OF SOUTHERN CALIFORNIA
}

\author{
STANFORD UNIVERSITY \\ UNIVERSITY OF TOKYO \\ UNIVERSITY OF UTAH \\ WASHINGTON STATE UNIVERSITY \\ UNIVERSITY OF WASHINGTON \\ * * * \\ AMERICAN MATHEMATICAL SOCIETY \\ CHEVRON RESEARCH CORPORATION \\ TRW SYSTEMS \\ NAVAL ORDNANCE TEST STATION
}

\footnotetext{
Mathematical papers intended for publication in the Pacific Journal of Mathematics should be typewritten (double spaced). The first paragraph or two must be capable of being used separately as a synopsis of the entire paper. It should not contain references to the bibliography. Manu. scripts may be sent to any one of the four editors. All other communications to the editors should be addressed to the managing editor, Richard Arens at the University of California, Los Angeles, California 90024.

50 reprints per author of each article are furnished free of charge; additional copies may be obtained at cost in multiples of 50 .
}

The Pacific Journal of Mathematics is published monthly. Effective with Volume 16 the price per volume (3 numbers) is $\$ 8.00$; single issues, $\$ 3.00$. Special price for current issues to individual faculty members of supporting institutions and to individual members of the American Mathematical Society: $\$ 4.00$ per volume; single issues $\$ 1.50$. Back numbers are available.

Subscriptions, orders for back numbers, and changes of address should be sent to Pacific Journal of Mathematics, 103 Highland Boulevard, Berkeley 8, California.

Printed at Kokusai Bunken Insatsusha (International Academic Printing Co., Ltd.), No. 6, 2-chome, Fujimi-cho, Chiyoda-ku, Tokyo, Japan.

PUBLISHED BY PACIFIC JOURNAL OF MATHEMATICS, A NON-PROFIT CORPORATION

The Supporting Institutions listed above contribute to the cost of publication of this Journal, but they are not owners or publishers and have no responsibility for its content or policies.

* Paul A. White, Acting Editor until J. Dugundji returns. 


\section{Pacific Journal of Mathematics}

\section{Vol. 16, No. $3 \quad$ BadMonth, 1966}

Gert Einar Torsten Almkvist, Stability of linear differential equations with

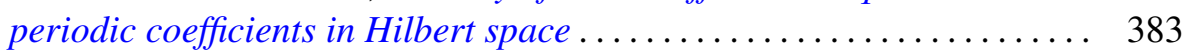

Richard Allen Askey and Stephen Wainger, A transplantation theorem for ultraspherical coefficients ................................ 393

Joseph Barback, Two notes on regressive isols .................. 407

Allen Richard Bernstein and Abraham Robinson, Solution of an invariant subspace problem of K. T. Smith and P. R. Halmos .............. 421

P. R. Halmos, Invariant subspaces of polynomially compact operators . . . . 433

Leon Bernstein, New infinite classes of periodic Jacobi-Perron algorithms.................................... 439

Richard Anthony Brualdi, Permanent of the direct product of matrices .... . 471

W. Wistar (William) Comfort and Kenneth Allen Ross, Pseudocompactness and uniform continuity in topological groups .................. 483

James Michael Gardner Fell, Algebras and fiber bundles . . . . . . . . . . . . 497

Alessandro Figà-Talamanca and Daniel Rider, A theorem of Littlewood and lacunary series for compact groups ..................... 505

David London, Two inequalities in nonnegative symmetric matrices...... 515

Norman Jay Pullman, Infinite products of substochastic matrices ........ 537

James McLean Sloss, Reflection and approximation by interpolation along

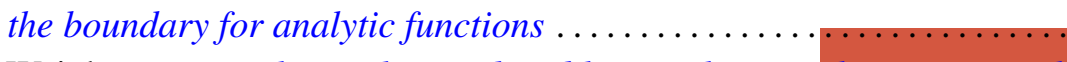

Carl Weinbaum, Visualizing the word problem, with an application to sixth groups................................... 